\title{
Comparative Two-Dimensional Gel Electrophoresis Maps for Promastigotes of Leishmania amazonensis and Leishmania major
}

\author{
Reynolds K. B. Brobey ${ }^{1}$, Fang C. Mei $^{2}$, \\ Xiaodong Cheng ${ }^{2}$ and Lynn Soong ${ }^{1}$
}

\author{
${ }^{1}$ Departments of Microbiology and Immunology, and Pathology, \\ Center for Biodefense and Emerging Infectious Diseases, Sealy \\ Center for Vaccine Development; ${ }^{2}$ Department of Pharmacology and \\ Toxicology; University of Texas Medical Branch, Galveston, TX, USA
}

\begin{abstract}
The outcome of Leishmania infections is determined by both the parasite species and the host genetic makeup. While much has been learned regarding immune responses to this parasite, our knowledge on parasite-derived factors is limited. The recent completion of the $L$. major and $L$. infantum genome sequence projects and concurrent advancement in proteomics technology would greatly accelerate the search for novel Leishmania proteins. Using a proteomics-based approach to study species-specific Leishmania proteins, we developed high-resolution, broad pH (3-10) two-dimensional gel electrophoresis (2-DE) separations to determine protein-expression profiles between highly infectious forms of the parasitic species L. amazonensis (New World) and L. major (Old World). Approximately 1,650 and 1,530 distinct protein spots were detected in the $L$. amazonensis and $L$. major gels, respectively. While a vast majority of the spots had similar distribution and intensity, a few were computationally defined as preferentially expressed in $L$. amazonensis in comparison to $L$. major, or vice versa. These data attest to the feasibility of establishing a 2-DE-based protein array for inter-species profiling of Leishmania proteins and provide the framework for future design of proteome studies of Leishmania. Key Words: Leishmania infections, gel electrophoresis, future studies.
\end{abstract}

Leishmaniasis is a vector-transmitted disease distributed throughout the world's tropical and subtropical regions. At least 20 Leishmania species give rise to a wide spectrum of clinical manifestations, ranging from self-healing skin ulcers or disfiguring mucosal lesions to fatal visceral infections. These varied clinical forms are primarily attributed to the host immune status and the species of the parasite involved; however, the determinants of species tissue tropism are largely unknown.

Leishmania parasites have a dimorphic life cycle, characterized by a free-living promastigote form and an intracellular amastigote form. Metacyclic promastigotes are transmitted to the mammalian host by the bite of phlebotomine sand flies. Once inside the host, the promastigotes are phagocytosed by macrophages, where they differentiate into nonmotile amastigotes. The promastigote form can be maintained in vitro in cell-free cultures, whereas the amastigote form can be isolated from mouse lesions, infected macrophages, or for some species, maintained in vitro by the differentiation of promastigotes using defined $\mathrm{pH}$ and temperature conditions. Given the technical difficulty in generating large quantities of amastigotes, we used late-stage stationary promastigotes in this study.

Received on 28 September 2005; revised 29 January 2006.

Address for correspondence: Dr. Lynn Soong. Department of Microbiology and Immunology, University of Texas Medical Branch, Medical Research Building 3.132, 301 University Blvd., Galveston, TX 77555-1070. Phone: (409) 772-8149. Fax: (409) 747-6869. Email: lysoong@utmb.edu

The Brazilian Journal of Infectious Diseases 2006;10(1):1-6. (C) 2006 by The Brazilian Journal of Infectious Diseases and Contexto Publishing. All rights reserved.
The Leishmania genome is spread over 36 chromosome pairs, ranging in size from 0.3 to $2.5 \mathrm{Mb}$, yielding a genome size of about $34 \mathrm{Mb}$. The genome sequence projects for $L$. major Friendlin (http://www.genedb.org/genedb/leish) and $L$. infantum (http://www.genedb.org/genedb/linfantum) have recently been completed. It is estimated that the Leishmania genome contains 8,272 protein-coding genes, only $36 \%$ of which can be ascribed a putative function [1]. Therefore, extensive studies are needed in the post-genomic era to search for the function of most ORFs. Of note, L. amazonensis and other members in the L. mexicana complex contain only 34 chromosomes (Chr), due to the fusion of chromosome 8 with 29 and 20 with 36 [2]. The biological significance of these genetic rearrangements remains unresolved; however, it is conceivable that these events influence protein expression and, consequently, the in-host behavior of the parasite.

Developmental regulation of gene expression in Leishmania is mediated almost exclusively at the posttranscriptional level, and it often involves the 3'-untranslated region of the RNA, which determines RNA stability and translational efficiency [2,3]. In addition, infectivity of the parasites is also dependent on extensive post-translational modifications [4]. Often mRNA abundance does not reflect protein levels in Leishmania, and methodologies to globally analyze gene expression at the protein level are especially useful for this organism. As an important human pathogen, Leishmania has been well studied and continues to be investigated in numerous areas, such as cell differentiation, host-parasite interactions, and for research on new drugs and vaccine targets. We feel that proteomics technology is a valuable tool to extend our understanding of diverse outcomes of Leishmania infection [5-7] and to accelerate the search for 
novel parasite proteins. Proteomics approaches to study Leishmania in general are at an early stage, and partial proteome maps based on two-dimensional gel electrophoresis (2-DE) have only recently been reported [8-10].

To further promote proteomics studies in Leishmania, we used high-resolution 2-DE to compare the steady-state protein expression of two Leishmania species, L. amazonensis and L. major, for the purpose of documenting global differences. The infectious promastigotes of both species were subjected simultaneously to 2 -DE protein profiling using broad $\mathrm{pH}$ (310). We looked for protein spots that are differentially expressed in either L. amazonensis or L. major by comparing spot intensity, and we also examined the feasibility of exploring this procedure to search for species-specific Leishmania proteins.

\section{Material and Methods}

Parasite infection of mice and in vitro cultivation. Promastigotes of L. amazonensis (MHOM/BR/77/LTB0016) and L. major (MRHO/SU/59/P/LV39) were cultured at $23^{\circ} \mathrm{C}$ in Schneider's Drosophila growth medium pH 7.0 (Life Technologies, Rockville, MD), supplemented with 20\% FBS (Sigma, St. Louis, MO), 2 mM L-glutamine, and $50 \mu \mathrm{g} / \mathrm{mL}$ gentamicin. To reduce potential variations that may be introduced during long-term cultivation of the parasites, all of the promastigotes employed in this study were recently recovered from infected $\mathrm{BALB} / \mathrm{c}$ mice and used before the $5^{\text {th }}$ generation in culture. To maintain parasite infectivity, female BALB/c mice (6-7 wk of age, Harlan Sprague-Dawley, Indianapolis, ID) were infected subcutaneously in the hind foot with $2 \times 10^{6}$ stationary-phase promastigotes; lesion-derived parasites were used to establish in vitro cultures. Mice were maintained under specific pathogen-free conditions, using protocols approved by the Animal Care and Use Committee of the University of Texas Medical Branch, Galveston, TX.

Protein preparation and 2-DE. Throughout the 2-DE procedures, promastigote samples of $L$. amazonensis and $L$. major were prepared side-by-side to minimize potential handling errors and technical variations. Protein extraction and 2-DE were performed following a modified protocol originally described by Lewis et al. [11]. Briefly, late-stage stationary promastigotes were washed three times in PBS and suspended in freshly prepared lysis buffer containing $7 \mathrm{M}$ urea, $2 \mathrm{M}$ thiourea, 2\% NP-40, 2\% DTT, and 2\% ampholines (pH 3-10, Pharmacia). Samples were incubated for $1 \mathrm{~h}$ at room temperature, with occasional vortexing, and then centrifuged for $15 \mathrm{~min}$ at $13,000 \mathrm{rpm}$. The protein supernatants were recovered and measured by a Non-Interfering Protein Assay ${ }^{\mathrm{TM}}$ (Geno Technology, Inc., St. Louis, MO), which gives linear responses in the presence of common laboratory agents (e.g., reducing agents, detergents, urea, etc). Soluble fractions of whole-cell extracts were adjusted to $150 \mu \mathrm{g} / 100 \mu \mathrm{L}$ and immediately frozen in aliquots at $-70^{\circ} \mathrm{C}$ until analysis.
For the first-dimension electrophoresis, a total of $300 \mu \mathrm{g}$ of protein was applied onto immobilized $\mathrm{pH}$ gradient (IPG) strips (17 cm, pH 3-10, Bio-Rad, Hercules, CA). After in-gel rehydration at $0 \mathrm{~V}$ for $12 \mathrm{~h}$, isoelectric focusing was performed at $500 \mathrm{~V}$ for $1 \mathrm{~h}, 1,000 \mathrm{~V}$ for $1 \mathrm{~h}$, and then at $8,000 \mathrm{~V}$ for $8 \mathrm{~h}$, using a Multiphor II electrophoresis unit and EPS 3500 XL power supply (Amersham, Piscataway, NJ). To promote efficient protein transfer from the first to second dimension, IPG strips were incubated in reducing buffer $(10 \mathrm{mM}$ DTT, $6 \mathrm{M}$ urea, $10 \%$ glycerol, $2 \%$ SDS, $50 \mathrm{mM}$ Tris, $\mathrm{pH} 6.8$ ) for $15 \mathrm{~min}$, followed by $15 \mathrm{~min}$ incubation in alkylation buffer $(100 \mathrm{mM}$ iodoacetamide, $6 \mathrm{M}$ urea, $10 \%$ glycerol, $2 \%$ SDS, $50 \mathrm{mM}$ Tris, $\mathrm{pH} 8.8$ ). Strips were then overlaid on freshly poured $10 \%$ Tricine-SDS gels $(18.3 \times 20 \mathrm{~cm})$ and sealed with agarose solution $(0.5 \%$ agarose, plus a few grains of bromophenol blue in a Tris-tricine cathode buffer). The protein standards, unstained Mark12, were purchased from Invitrogen (Carlsbad, CA). Gel electrophoresis was carried out at $75 \mathrm{~V}$ for $1 \mathrm{~h}$, and then at $140 \mathrm{~V}$ until the dye front reached the bottom of the gel $(\sim 18 \mathrm{~h})$. Gels were silver-stained simultaneously under identical conditions using a specially designed, multi-chambered plastic staining tray, and freshly prepared silver reagents, as previously described [12].

Image acquisition and spot comparisons. Stained gels were scanned at a resolution of 100 microns for $1100 \mathrm{~ms}$ using a Perkin-Elmer ProXPRESS ${ }^{\mathrm{TM}}$ Proteomic Imaging System (Boston, MA). The exposure time was adjusted to achieve a value of $\sim 55,000-63,000$ pixel intensity. The images were subsequently analyzed using Nonlinear Dynamics Progenesis v.2003.03 software (Newcastle upon Tyne, UK). For the purpose of this experiment, each set of gel replicates for both the L. amazonensis and L. major samples was combined into average gels, which represented spots that were reproducibly present on both sets of the replicate gels. The software automatically selected the gel with the greatest number of spots as the image for the reference gel and added unmatched spots from the other gels to this image to give a comprehensive reference gel for matching spots on the different gels. The spots detected automatically by the software were visually inspected. Spot filtering and editing were performed manually to remove artifacts and to correct for spots that did not split correctly or were not detected by the software's automatic spot detection process. After the filtering and editing steps, the average gels were recreated, and the spots on the average gels were matched, using the comprehensive reference gel as a map to link the spots on the different gels. The software subtracted a background value for each spot based on the entire surface area of the gel. Spot volumes were normalized, based on the total volume of all of the spots on each gel, and both of these operations were performed recursively every time spots were edited on any of the gels. Finally, a 2-fold change criterion was applied for comparison of spots on the L. amazonensis and L. major average gels. Each spot that met 
this criterion was then visually verified to see if it was correctly detected, and the accuracy of the matching of the spots between the replicate sets was confirmed.

\section{Results}

Generation of 2-DE maps for promastigotes of L. amazonensis and L. major. A global approach, such as 2-DE protein profiling, is a useful tool to define the protein repertoire of microorganisms. While substantial literature on proteome profiling for yeast and bacteria exists $[13,14]$, there are only a few reports for profiling parasite proteins at the global level $[8,10,15,16]$. The purpose of our study was to establish 2-DE as a tool to study protein expression patterns of $L$. amazonensis and L. major, with an ultimate goal of employing proteomics technologies to search for species-specific Leishmania proteins. We used axenic promastigotes in this study, since the promastigote stage of Leishmania is easily cultured in large quantities in vitro, when compared to the amastigotes.

Soluble proteins were prepared from stationary-phase promastigotes that were recently recovered $(\leq 5$ in vitro passages) from BALB/c mice by solubilizing parasite wholecell lysate with lysis buffer containing urea and NP-40. Proteins were separated over $\mathrm{pH}$ 3-10 on the first dimension, followed by a second dimension run on $10 \%$ Tricine-SDS gels. The reproducibility of sample preparations, gel electrophoresis and the subsequent staining procedures, was verified by performing replicates of experiments run on different batches of parasites.

Representative 2-DE maps of L. amazonensis and L. major are shown in Figure 1. After thorough automated and manual editing to correct for artifacts (see Material and Methods), the image analysis confidently detected approximately 1,650 spots for L. amazonensis (Figure 1A) and 1,530 spots for $L$. major (Figure 1B), which represented $8 \%$ fewer spots for $L$. major than those for L. amazonensis. Overall, there was a striking similarity in protein distribution and positioning between the two Leishmania species, with the majority of the proteins ranging from $\mathrm{p} I 4$ to $\mathrm{p} I 7$. An enrichment of protein spots within the $\mathrm{p} I$ 4-7 has also been reported for other Leishmania species $[10,17]$. With the exception of the extreme acidic ends of the gels, where resolution was suboptimal, spots were generally well separated, and substantial numbers of similarly placed spots could be visualized manually.

Quantitation of the expressed spots. The average gels of $L$. amazonensis and L. major were matched densitometrically by using a computerized program that normalized the volume of each spot against the total valid spots in each gel. Spots with a 2-fold or greater difference in intensity in L. amazonensis over that in $L$. major, or vice-versa, were documented as differentially expressed. These spots are marked with circles in Figure 1 and are listed in Table 1. Unless indicated, unlabeled

Figure 1. Detection of Leishmania promastigote proteins via 2-DE. Soluble proteins of whole parasite lysates of L. amazonensis (A) and L. major (B) were run on pH 3-10 IPG strips $(17 \mathrm{~cm})$ and then separated on $10 \%$ Tricine-SDS gels $(18 \times 20 \mathrm{~cm})$. Gels were silverstained simultaneously. Spots that were confirmed to show a 2 -fold or greater increase in L. amazonensis over $L$. major, or vice-versa, were marked with circles in the corresponding image. The numbers shown correlate with spot reference numbers assigned via computer software in Table 1. Spots that were found in the L. amazonensis gel, but were barely detected in L. major, were also circled in both gels.

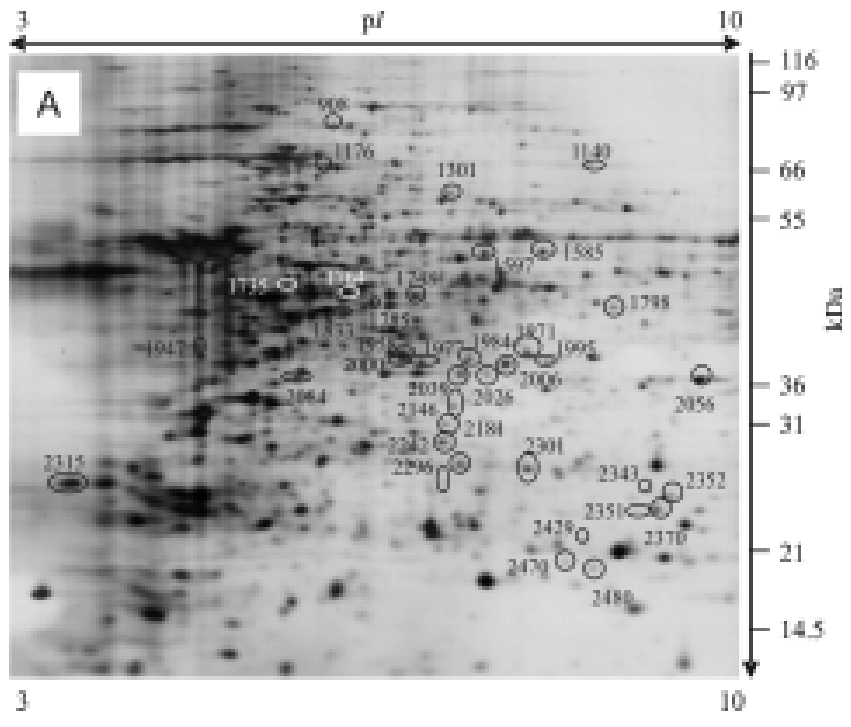

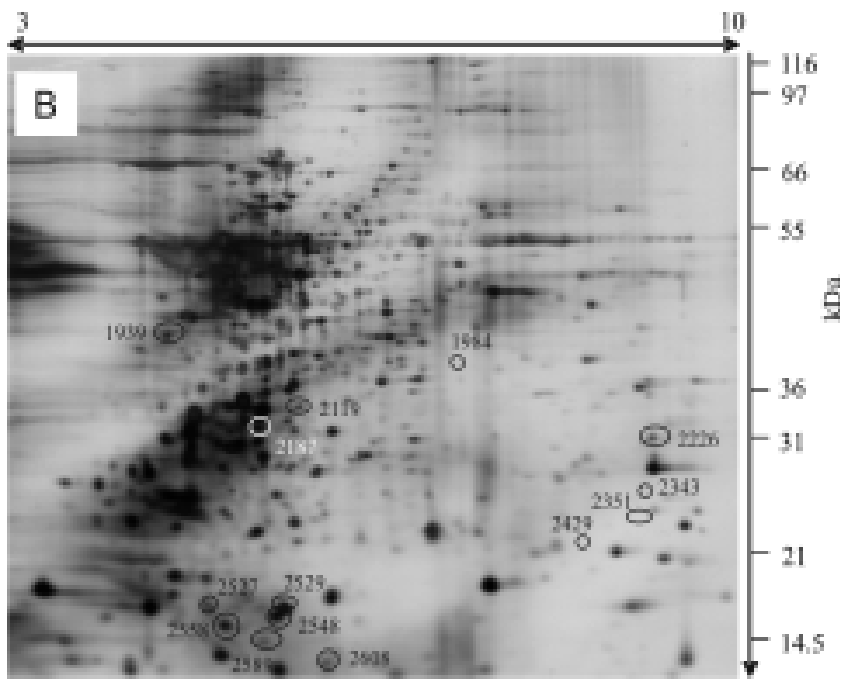


Figure 2. Close-up views of differentially or specifically expressed protein spots in the promastigotes of $L$. amazonensis and L. major. Images in A, B and $\mathrm{C}$ are representative areas of spots separated under $\mathrm{pH}$ 8.3-10, 6.78.0, and 4.6-6.5 were extrapolated from Fig. 1. Spots that showed differences in abundance and/or were assumed to be specifically expressed are marked in circles, and the numbers correlate with spot reference numbers listed in Table 1.
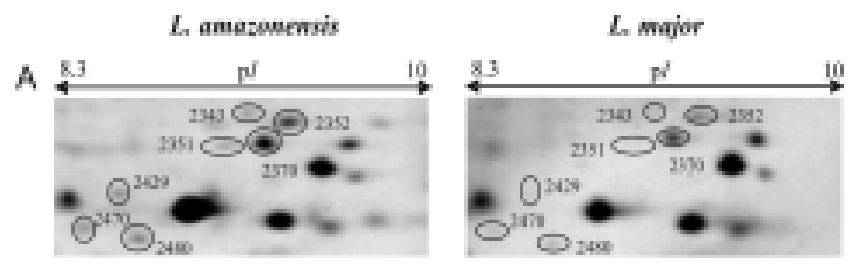

B
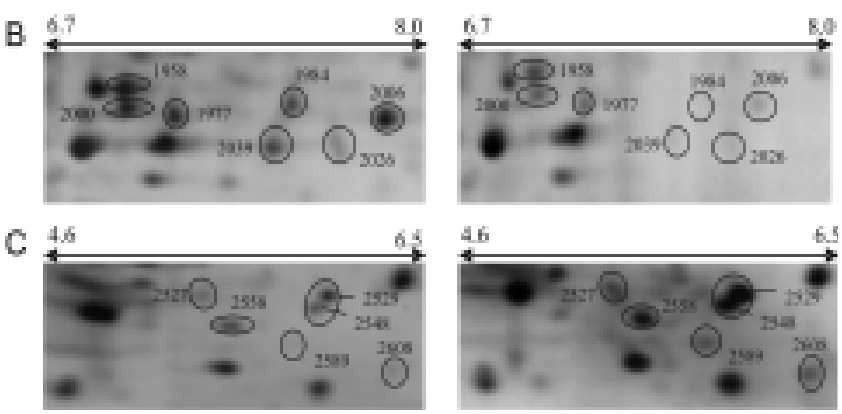

spots were either considered background or displayed no significant differences between the two Leishmania species. Most L. amazonensis protein spots with an increased intensity relative to L. major had pIs in the alkaline region (Figure 1A), whereas most of the L. major proteins with enhanced expression had acidic to neutral pIs (Figure 1B). A close-up view of selected gel areas is shown in Figure 2. The software also recognized spots that were readily detectable in the $L$. amazonensis gel, but barely visible in the corresponding locations in the L. major gel. For example, spots with reference numbers 2343, 2351, 2429, and 1984 were apparently detected in L. amazonensis, but not in L. major (Fig. 2A and 2B). Overall, 47 spots were confidently determined to be differentially expressed between L. amazonensis and L. major (Table 1), although these spots ultimately need to be confirmed by mass spectrometry identification.

\section{Discussion}

The completion of genome sequencing initiatives for numerous pathogenic microbial species has given the scientific community insight into the disease process for many of them. Comparative genomics has been utilized to comprehend the changes in genetic constitution associated with a given phenotype (e.g., bacterial virulence) $[13,18]$. Microarray technologies at the transcriptome level provide additional information for selecting relevant molecules for
Table 1. Forty seven spots differently expressed in promastigotes of L. amazonensis $(L a)$ or in L. major were confidently detected from 2D gels ( $\mathrm{pH} \mathrm{3-10;} \mathrm{see} \mathrm{Figure} \mathrm{1)}$ using Nonlinear Dynamics Progenesis v.2003.03 software.

\begin{tabular}{|c|c|c|c|c|}
\hline & Ref. spot $^{\mathrm{a}}$ & $\begin{array}{l}\text { Fold } \\
\text { difference }^{b}\end{array}$ & pI & M.wt. \\
\hline \multirow[t]{37}{*}{$\overline{L a}$} & $1984^{c}$ & - & 7.5 & 39.7 \\
\hline & $2429^{c}$ & - & 8.5 & 21.3 \\
\hline & 1798 & 16.0 & 8.7 & 45.0 \\
\hline & 2039 & 13.2 & 7.5 & 37.7 \\
\hline & 2146 & 10.5 & 7.4 & 33.7 \\
\hline & 2301 & 7.2 & 8.0 & 26.7 \\
\hline & 1748 & 7.1 & 7.0 & 46.7 \\
\hline & 2470 & 6.8 & 8.4 & 20.1 \\
\hline & 1301 & 6.0 & 7.3 & 60.9 \\
\hline & 1995 & 5.1 & 8.2 & 39.4 \\
\hline & 1764 & 4.3 & 6.5 & 46.1 \\
\hline & 2006 & 4.1 & 7.8 & 39.2 \\
\hline & 2296 & 4.1 & 7.4 & 27.0 \\
\hline & 2352 & 4.0 & 9.2 & 24.4 \\
\hline & 908 & 4.0 & 6.4 & 83.9 \\
\hline & 1947 & 3.9 & 5.2 & 40.9 \\
\hline & 1977 & 3.8 & 7.1 & 39.9 \\
\hline & 1585 & 3.8 & 8.1 & 51.4 \\
\hline & 2480 & 3.7 & 8.6 & 19.7 \\
\hline & $2351^{\mathrm{c}}$ & - & 8.9 & 24.4 \\
\hline & $2343^{c}$ & - & 9.0 & 24.8 \\
\hline & 1971 & 3.5 & 8.0 & 40.1 \\
\hline & 2056 & 3.3 & 9.5 & 37.1 \\
\hline & 1176 & 3.1 & 6.3 & 66.4 \\
\hline & 2000 & 3.1 & 6.9 & 39.3 \\
\hline & 1140 & 2.8 & 8.5 & 68.1 \\
\hline & 1597 & 2.8 & 7.6 & 51.0 \\
\hline & 1785 & 2.8 & 6.7 & 45.6 \\
\hline & 2242 & 2.8 & 7.3 & 29.4 \\
\hline & 2084 & 2.7 & 6.0 & 36.4 \\
\hline & 2181 & 2.7 & 7.3 & 31.7 \\
\hline & 2370 & 2.6 & 9.1 & 23.0 \\
\hline & 1833 & 2.5 & 6.4 & 44.5 \\
\hline & 1735 & 2.5 & 5.9 & 47.1 \\
\hline & 2315 & 2.5 & 4.1 & 25.9 \\
\hline & 2026 & 2.2 & 7.7 & 38.1 \\
\hline & 1958 & 2.1 & 6.9 & 39.6 \\
\hline \multirow[t]{10}{*}{ L.major } & 2548 & -9.5 & 5.8 & 15.5 \\
\hline & 2589 & -8.4 & 5.7 & 14.0 \\
\hline & 2529 & -5.0 & 5.9 & 17.4 \\
\hline & 2608 & -3.9 & 6.3 & 13.9 \\
\hline & 2187 & -3.4 & 5.7 & 31.4 \\
\hline & 2226 & -3.0 & 9.1 & 30.7 \\
\hline & 2527 & -2.5 & 5.3 & 17.9 \\
\hline & 1939 & -2.5 & 4.7 & 41.3 \\
\hline & 2558 & -2.2 & 5.5 & 16.4 \\
\hline & 2119 & -2.2 & 6.1 & 34.8 \\
\hline
\end{tabular}

pI, isoeletric point; M.wt., molecular weight. ${ }^{a}$ Reference numbers were assigned digitally to each spot prior to spots editing, and shown are corresponding protein spots circled in Figure $1 \mathrm{~A}$ or $1 \mathrm{~B} .{ }^{\mathrm{b}}$ Fold of differences in spot intensity are shown in positive ( $L a$ above L.major)

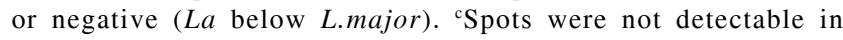
corresponding positions in L.major gels. 
further analysis, aimed at validating whether the presence or absence of a gene correlates with a biological function. While these research efforts are still of prime importance for complex pathogens such as Leishmania, extensive post-translational modifications associated with certain Leishmania proteins [4] suggest that data transfer between the transcriptome and the proteome is not straightforward. Emphasis needs to be placed at the protein level. In this study, we developed highresolution, reproducible $2-\mathrm{DE}$ protein arrays to study the protein expression pattern of two geographically and immunologically distinct Leishmania species, L. amazonensis in the New World and L. major in the Old World [6,7].

At present, 2-DE, facilitated by the use of both medium and narrow range IPG strips in the first dimension, is the method of choice for simultaneously separating and quantifying a complex mixture of proteins [19]. Comparative proteomics has been successful in determining potential virulence markers in human pathogens, such as Mycobacteria species and Helicobacter pylori $[13,20]$. By contrast, proteomes of many parasitic protozoa remain to be unraveled, but the near completion of genome sequencing for several apicomplexans, coupled with the improved cell culture and lysis, protein separation and visualization techniques that are currently available, will accelerate proteomic studies for these organisms.

We performed a side-by-side, 2-DE comparative analysis of L. amazonensis and L. major promastigotes using $17-\mathrm{cm}$ IPG strips at a broad $\mathrm{pH}$ range $(\mathrm{pH} 3-10)$. We resolved about 1,650 protein spots for L. amazonensis and 1,530 protein spots for L. major, representing approximately $20 \%$ of the estimated proteins in Leishmania genome projects [1]. The number of spots resolved in this study is much higher than a previous report [17], but lower than the 3,700 spots resolved in L. major gels using a combination of medium- and narrow-range IPG strips [8]. The experimental procedures used here for complex organisms like Leishmania enabled us to accurately compare protein distribution and positioning between the two species. The close match in terms of protein distribution and positioning for the two species used here is quite obvious, but inter-species variability in terms of spot abundance and positioning is also evident. Although protein identification will be needed to establish the authenticity of these variable spots, the gel-to-gel reproducibility and improved spot resolution achieved here provide a reliable approach for designing future proteome studies aimed at enriching protein fractions for defined protein identification.

Our study demonstrates that 2-DE, when used under optimal conditions, has the capacity to resolve Leishmania proteins and corroborates findings in previous studies on the readiness of Leishmania for proteomics technology. Although the 2-DE system is a powerful and reliable tool, it has several technical limitations, especially the inefficiency in adequately resolving membrane proteins, which are major targets for therapeutic compounds $[21,22]$. To obtain a complete representative proteome, other technologies must be sought in a complementary fashion. For example, a direct MS-based approach has been used successfully in Plasmodium falciparum [23]. A combination of the narrow-pH 2-DE system and Western blot detection system would be a powerful approach for identifying Leishmania proteins that are preferentially recognized by sera for human patients [24], as well as healing versus non-healing animals (our unpublished results).

Concluding remarks. Despite the rich knowledge of differential host immune responses to cutaneous infection with these two parasites, very little is known about how parasite-derived factors/proteins contribute to the speciesspecific outcome of the disease. Establishing a global methodology for protein profiling will accelerate the search for such novel Leishmania proteins. Our study demonstrates that high-resolution, reproducible 2-DE is a powerful tool to document protein expression profiles of L. amazonensis and L. major. The reproducibility of the gel-to-gel comparison achieved in this study will encourage studies to identify some of these differentially expressed proteins, and, in conjunction with immunological assays, may lead to the identification of essential parasite proteins and the development of new Leishmania control strategies.

\section{Acknowledgments}

We are grateful to Drs. Zheng Wu and M. Jawad Pashmi of the Proteomics Core Laboratory-UTMB for documenting the gel images and Travis Young for technical assistance in 2DE preparations; we also thank Mardelle Susman and Joseph Masterson for comments on the manuscript. This study was supported in part by a John W. McLaughlin Fellowship to R. B. Brobey and NIH grant AI43003 to L. Soong.

\section{Abbreviations}

Abbreviations used in this manuscript: 2-DE, twodimensional gel electrophoresis; IPG, immobilized $\mathrm{pH}$ gradient; $\mathrm{p} I$, isoelectric point.

\section{References}

1. Ivens A.C., Peacock C.S., Worthey E.A., et al. The genome of the kinetoplastid parasite, Leishmania major. Science 2005;309:436-42.

2. Stiles J.K., Hicock P.I., Shah P.H., Mead J.C. Genomic organization, transcription, splicing and gene regulation in Leishmania. Ann Trop Med Parasitol 1999;93:781-807.

3. Rochett A., McNicoll F., Girard, J., et al. Characterization and developmental gene regulation of a large gene family encoding amastin surface proteins in Leishmania spp. Mol Biochem Parasitol 2005; 140:205-20.

4. Ilgoutz SC, McConville M.J. Function and assembly of the Leishmania surface coat. Int J Parasitol 2001;31:899-908. 
5. Soong L., Chang C.H., Sun J., et al. Role of CD4+ T-cells in pathogenesis associated with Leishmania amazonensis infection. J Immunol 1997; 158:5374-83.

6. Ji J., Sun J., Soong L. Impaired expression of inflammatory cytokines and chemokines at early stages of infection with Leishmania amazonensis. Infect Immun 2003; $71: 4278-88$.

7. Ji J., Masterson J., Sun J., Soong, L. CD4 ${ }^{+} \mathrm{CD} 25^{+}$regulatory Tcells restrain pathogenic responses during Leishmania amazonensis infection. J Immunol 2005; 174:7147-53.

8. Drummelsmith J., Brochu V., Girard I., et al. Proteome mapping of the protozoan parasite Leishmania and application to the study of drug targets and resistance mechanisms. Mol Cell Proteomics 2003;2:146-55.

9. El Fakhry Y., Ouellette M., Papadopoulou B. A proteomic approach to identify developmentally regulated proteins in Leishmania infantum. Proteomics 2002;2:1007-17.

10. Gongora R., Acestor N., Quadroni M., et al. Mapping the proteome of Leishmania Viannia parasites using two-dimensional polyacrylamide gel electrophoresis and associated technologies. Biomedica 2003;23:15360.

11. Lewis T.S., Hunt J.B., Aveline L.D., et al. Identification of novel MAP kinase pathway signaling targets by functional proteomics and mass spectrometry. Mol Cell 2000;6:1343-54.

12. Bassam B.J., Caetano-Anolles G., Gresshoff P.M. Fast and sensitive silver staining of DNA in polyacrylamide gels. Anal Biochem 1991; 196:80-3.

13. Cordwell S.J., Nouwens A.S., Walsh B.J. Comparative proteomics of bacterial pathogens. Proteomics 2001;1:461-72.

14. Covert B.A., Spencer J.S., Orme I.M., Belisle J.T. The application of proteomics in defining the T-cell antigens of Mycobacterium tuberculosis. Proteomics 2001;1:574-86.
15. Bromley E., Leeds N., Clark J., et al. Defining the protein repertoire of microneme secretory organelles in the apicomplexan parasite Eimeria tenella. Proteomics 2003;3:1553-61.

16. Florens L., Washburn M.P., Raine J.D., et al. A proteomic view of the Plasmodium falciparum life cycle. Nature 2002;419:520-6.

17. Acestor N., Masina S., Walker J., et al. Establishing twodimensional gels for the analysis of Leishmania proteomes. Proteomics 2002; 2:877-9.

18. Doumith M., Cazalet C., Simoes N., et al. New aspects regarding evolution and virulence of Listeria monocytogenes revealed by comparative genomics and DNA arrays. Infect Immun 2004; 72:1072-83.

19. Rabilloud T. Two-dimensional gel electrophoresis in proteomics: old, old fashioned, but it still climbs up the mountains. Proteomics 2002;2:3-10.

20. Bumann D., Aksu S., Wendland M., et al. Proteome analysis of secreted proteins of the gastric pathogen Helicobacter pylori. Infect Immun 2002;70:3396-403.

21. Legare D., Cayer S., Singh A.K., et al. ABC proteins of Leishmania. J Bioenerg Biomembr 2001;33:469-74.

22. El Fadili K., Messier N., Leprohon P., et al. Role of the ABC transporter MRPA (PGPA) in antimony resistance in Leishmania infantum axenic and intracellular amastigotes. Antimicrob Agents Chemother 2005;49:1988-93.

23. Lasonder E., Ishihama Y., Andersen J.S., et al. Analysis of the Plasmodium falciparum proteome by high-accuracy mass spectrometry. Nature 2002;419:537-42.

24. Kamoun-Essghaier S., Guizani I., Strub J.M., et al. Proteomic approach for characterization of immunodominant membrane-associated 30- to 36-kilodalton fraction antigens of Leishmania infantum promastigotes, reacting with sera from Mediterranean visceral leishmaniasis patients. Clin Diagn Lab Immunol 2005; 12:310-20. 